\title{
Cell-Assisted Lipotransfer for Facial Lipoatrophy: Efficacy of Clinical Use of Adipose-Derived Stem Cells
}

\author{
Kotaro Yoshimura, MD, ${ }^{*}$ Katsujiro Sato, MD ${ }^{\dagger}$ Noriyuki Aoi, MD, ${ }^{*}$ Masakazu Kurita, MD ${ }^{\ddagger}$ \\ Keita Inoue, MD, ${ }^{*}$ Hirotaka Suga, MD, ${ }^{*}$ Hitomi Eto, MD,${ }^{*}$ Harunosuke Kato, MD, ${ }^{*}$ \\ Toshitsugu Hirohi, MD, ${ }^{\S}$ AND KiYONORi Harit, MD
}

BACKGROUND Lipoinjection is a promising treatment, but its efficacy in recontouring facial lipoatrophy remains to be established.

OBJECTIVE The objective was to evaluate the efficacy and adverse effects of lipoinjection and supplementation of adipose-derived stem/stromal cells (ASCs) to adipose grafts.

METHODS To overcome drawbacks of autologous lipoinjection, we have developed a novel strategy called cell-assisted lipotransfer (CAL). In CAL, stromal vascular fraction containing ASCs was freshly isolated from half of an aspirated fat sample and attached to the other half of aspirated fat sample with the fat acting as a scaffold. This process converts relatively ASC-poor aspirated fat into ASC-rich fat. We performed conventional lipoinjection (non-CAL; $n=3$ ) or CAL $(n=3)$ on six patients with facial lipoatrophy due to lupus profundus or Parry-Romberg syndrome.

RESULTS All patients obtained improvement in facial contour, but the CAL group had a better clinical improvement score than did the non-CAL patients, although the difference did not reach statistical significance $(p=.11)$. Adipose necrosis was found in one non-CAL case who took perioperative oral corticosteroids.

CONCLUSION Our results suggest that CAL is both effective and safe and potentially superior to conventional lipoinjection for facial recontouring.

The authors have indicated no significant interest with commercial supporters.

$\mathrm{F}$ acial lipoatrophy is a disfiguring and socially disabling problem that accompanies several inherited and acquired diseases (see Ascher et al. ${ }^{1}$ for review). Lupus erythematosus profundus and scleroderma en coup de sabre (en coup de sabre morphea) frequently give rise to facial lipoatrophy, which is often the most problematic manifestation of the disease for the patients even though they require medication, such as oral corticosteroids, to suppress other symptoms associated with the systemic morbidities. Facial lipoatrophy is also seen in patients with human immunodeficiency virus (HIV) infection and Parry-Romberg syndrome (hemifacial progressive atrophy or idiopathic hemifacial atrophy), which involves not only facial lipoatrophy but also bony defects. ${ }^{2}$ Thus far there is no medical treatment to correct facial lipoatrophy.

Lipoinjection has been used for treating lipoatrophy such as that associated with HIV infection with moderate success, ${ }^{3,4}$ while microsurgical tissue transfer has been a standard surgical treatment for recontouring facial defects seen in Parry-Romberg syndrome. However, the surgical procedure leaves conspicuous scars on the face and the donor site. ${ }^{5,6}$ Autologous lipoinjection is a promising treatment for soft tissue augmentation because there is no associated incision scar or complications associated with foreign materials. Although

*Department of Plastic Surgery, University of Tokyo School of Medicine, Tokyo; ${ }^{\dagger}$ Cellport Clinic Yokohama, Yokohama; ${ }^{\ddagger}$ Department of Plastic Surgery, Kyorin University School of Medicine, Tokyo; ${ }^{\S}$ Ritz Cosmetic Surgery Clinic Tokyo, Tokyo, Japan 
many innovative efforts to refine autologous lipoinjection have been reported, ${ }^{7-14}$ problems such as unpredictability and a low rate of graft survival due to partial necrosis remain.

To overcome these problems, we have developed a novel strategy, called cell-assisted lipotransfer (CAL; Figure 1).

Since a fraction of stromal cells derived from the adipose tissue was found to have the capacity to differentiate into various cell lineages, ${ }^{15}$ the stromal progenitor cells have been designated "adipose-derived stem/stromal cells" (ASCs) and are expected to become a valuable tool in a wide range of cell-based therapies. The therapeutic concept of CAL was described in a previous report of our preclinical studies. ${ }^{16}$ In CAL, ASC-poor fat is converted to ASC-rich fat by supplementing with cells freshly isolated from the adipose tissue during preparation of the injectable material (Figure 1). We report the preliminary clinical results of patients who underwent CAL for recontouring of acquired facial lipoatrophy. This is the first report of the clinical use of ASCs for soft tissue augmentation of the face.

\section{Cell-Assisted Lipotransfer (CAL)}

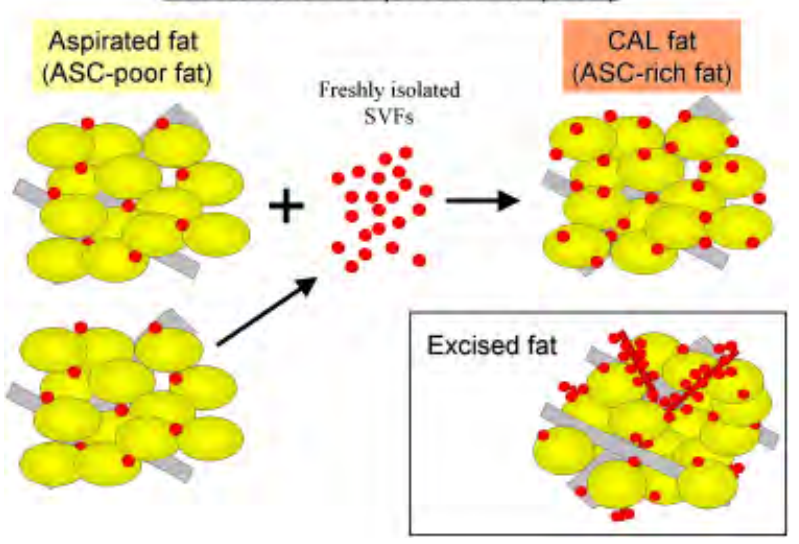

Figure 1. Scheme of CAL. Half of the volume of the aspirated fat is processed for isolation of the SVF. During the isolation process, the other half of the aspirated fat is prepared for grafting. Freshly isolated SVF-containing ASCs are attached to the aspirated fat with the fat acting as a living scaffold. Finally, the SVF-supplemented fat is injected into the target sites. Thus, aspirated fat, which is originally relatively poor in ASCs, is converted to ASC-rich fat.

\section{Materials and Methods}

\section{Patients}

We analyzed the outcomes of six patients with facial lipoatrophy who underwent lipoinjection for recontouring facial defects: three patients underwent conventional lipoinjection (non-CAL), while three patients underwent CAL. No patient underwent more than one CAL or non-CAL procedure. Informed consent was obtained from all patients. The clinical trial protocol conformed to the guidelines of the 1975 Declaration of Helsinki and was approved by individual institutional review boards.

One of the six patients had been diagnosed with Parry-Romberg syndrome; the other five patients had been diagnosed with lupus erythematosus profundus by their dermatologists. Two of the lupus cases (one in the non-CAL group and one in the CAL group) were taking oral steroids (predonisolone) at the time of surgery. Severity grading of facial lipoatrophy was determined according to the grading scale (graded 1 to 5,5 being the most severe) developed by the Facial Lipoatrophy Panel. ${ }^{1}$ Patients were evaluated between 9 and 13 months as described later. One of the three patients in each group was male, and the patient's ages ranged from 33 to 55 years (mean age \pm SD, non-CAL $46.3 \pm 7.8$, CAL $38.7 \pm 8.1$ ). The mean volume of injected fat was $133 \pm 104 \mathrm{~mL}$ in the non-CAL group and $100 \pm 10 \mathrm{~mL}$ in the CAL group. Patient data are summarized in Table 1.

\section{Surgical Techniques}

Before suctioning, the abdominal wall was infiltrated with saline solution with diluted epinephrine $(0.001 \%)$ with the patient under general anesthesia. Adipose tissue was suctioned using a cannula with 2.5-mm inner diameter and a conventional liposuction machine. In the CAL group, about a half of the collected liposuction aspirate was used for isolation of the stromal vascular fraction (SVF). The SVF was isolated from both the adipose portion and the fluid portion of liposuction aspirates as previously 
TABLE 1. Summary of Patients' Data

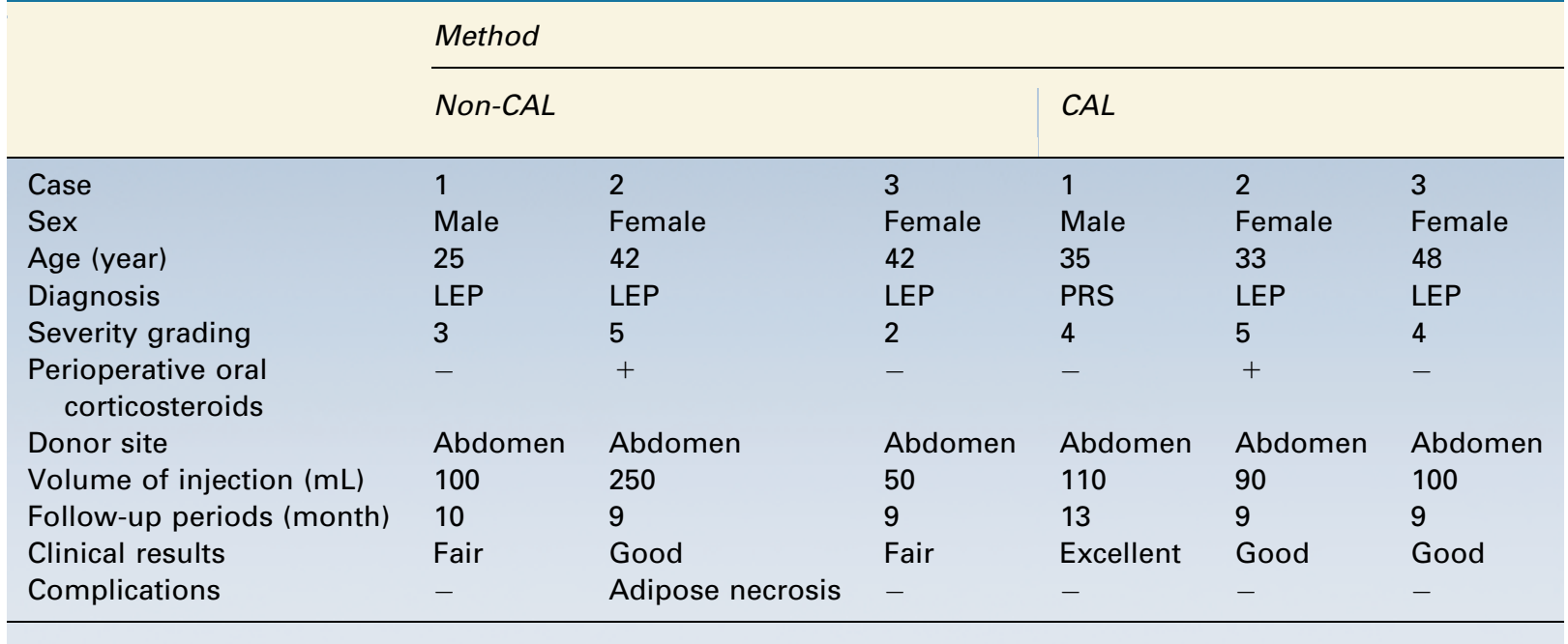

LEP, lupus erythematosus profundus; PRS, Parry-Romberg syndrome.

described. ${ }^{17}$ In brief, the adipose portion of liposuction aspirates was digested with $0.075 \%$ collagenase in buffered saline for 30 minutes on a shaker at $37^{\circ} \mathrm{C}$. Mature adipocytes and connective tissue were separated from the SVF containing ASCs by centrifugation $(800 \times g, 10$ minutes), and then rinsed three times with buffered saline. The fluid portion was centrifuged ( $800 \times g, 5$ minutes), and the pellets were resuspended in hypotonic water to lyse erythrocytes. The cell processing procedure took about 90 minutes. During the processing period, the other half of lipoaspirates was harvested as graft material. The surgery was performed in a sterile operating room, and the cell isolation was performed in a sterile cell processing room.

The adipose portion of liposuction aspirates was centrifuged at $700 \times g$ for 3 minutes without washing and put into a metal jar $(500 \mathrm{~mL})$ that was placed in water with crushed ice. In the non-CAL group, centrifuged fat was injected without SVF supplementation. In the CAL group, the fresh SVF isolated from both the adipose and the fluid portion was added to the graft material and, after gentle mixing and waiting for 10 to 15 minutes for cell adherence to the aspirated fat, the cellsupplemented fat was then put into an injection syringe.
For the injection syringe, a $10-\mathrm{cm}^{3}$ inflator (LeVeen, Boston Scientific Corp., Boston, MA) was used because they are screw-type syringes (with a threaded plunger) and threaded connections that fit both the connecting tube and the needle, to allow for precise control during injection. An 18-gauge needle (25 or $60 \mathrm{~mm}$ long) was used for lipoinjection and inserted in several layers (the subcutaneous fatty layer and the muscle layer) and directions to achieve diffuse distribution of the graft materials. Quantity of transplanted adipose tissue was determined by trying to overcorrect by about $20 \%$.

\section{Evaluation of Clinical Improvement}

Photographs of each patient were taken before and after treatment with a high-resolution digital camera (Model D30, Canon, Tokyo, Japan). The percentage of volumetric improvement of facial defects was determined via evaluation of preoperative and postoperative photographs by four certified plastic surgeons blinded to clinical data. The mean data of the volumetric improvement of each patient were classified into four categories: excellent $(80 \%$ improvement or better), good (60\% to less than $80 \%$ improvement), fair ( $40 \%$ to less than $60 \%$ improvement), and poor (less than $40 \%$ improvement). 


\section{Results}

The transplantation of adipose tissue was successfully performed in all cases. Subcutaneous bleeding was seen on some parts of the face and resolved within 1 to 2 weeks. Patients usually recovered from postoperative swelling at about 4 weeks. Transplanted adipose tissue was gradually absorbed during the first 2 postoperative months. The volume continued to be reduced in the non-CAL group, while it showed a minimal change thereafter in the CAL group. Patient data are summarized in Table 1, and photographs of two representative cases in each group are shown in Figures 2 through 5. All patients showed cosmetic improvements, but the degree varied among patients. CAL had a better clinical improvement score than non-CAL, although the difference did not reach statistical significance $(p=.11)$.

The reconstructed tissue was generally soft and with natural texture, although some fibrous tissues that had existed before surgery appeared to remain. In one case of the non-CAL group (non-CAL Case 2; Figure 3), redness and fluctuation were detected on the left cheek 1 month after surgery and necrotized adipose fluids were drained through a small incision. We cultured this fluid, but did not detect any bac- teria. In other cases, no adverse effects were seen and any nodules or cysts were not palpable.

\section{Discussion}

In the CAL strategy, autologous ASCs are used to enhance angiogenesis, improve the survival rate of grafts, and reduce postoperative atrophy. In CAL, half the volume of the aspirated fat is processed for isolation of the SVF-containing ASCs (Figure 1).

During the isolation process, the other half of the aspirated fat is prepared for grafting. Freshly isolated SVF, which contains ASCs as well as vascular endothelial cells, pericytes, blood cells (WBCs and RBCs), and other cells as previously described, ${ }^{17}$ is attached to the aspirated fat with the fat acting as a living scaffold before transplantation. Finally, the progenitorenriched fat tissue is injected into the target sites.

Using our technique, aspirated fat was harvested with a relatively large-sized suction cannula, centrifuged at $700 \times g$, and kept cool until transplantation. We believe that centrifugation of aspirated fat substantially affects survival rate of injected fat because centrifugation at $1,200 \times g$ experimentally decreases the fat volume by $30 \%$, damages $12 \%$ of the adipocytes, and $0 \%$ of the ASCs, which concentrates
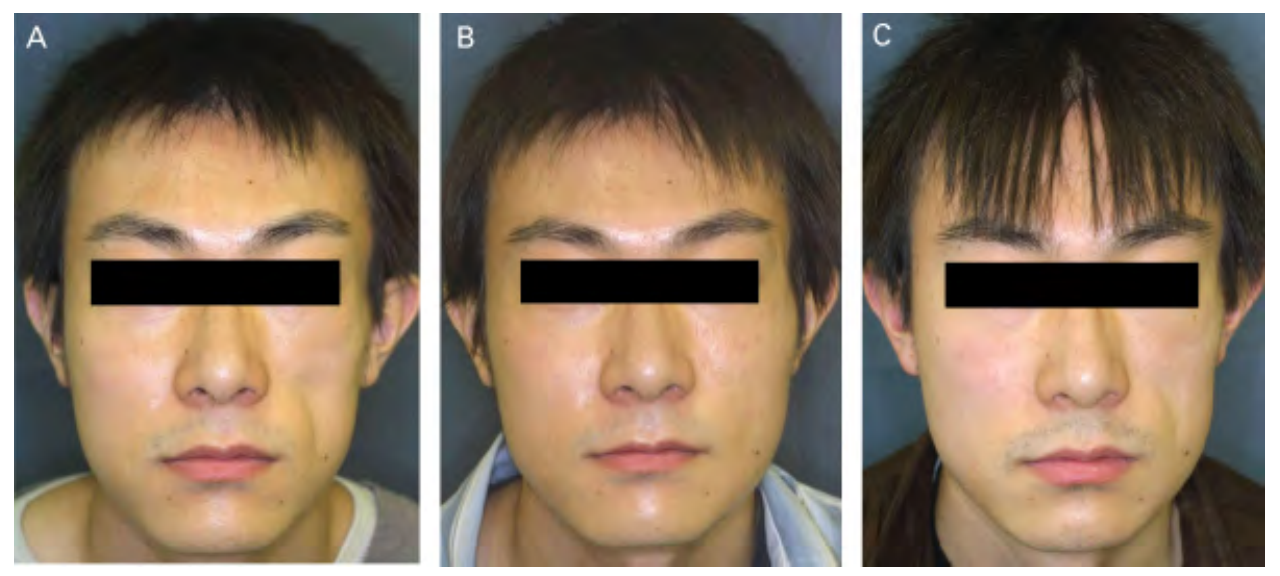

Figure 2. Clinical views of non-CAL Case 1 (a 25-year-old man): preoperative views (Grade 3 lipoatrophy; A) and postoperative views at 1 month (B) and at 10 months (C). The patient was diagnosed as LEP at the age of 21 years and started oral corticosteroid therapy. He stopped the medication 1 year ago and has experienced significant change in the past 12 months. Conventional lipoinjection $(100 \mathrm{~mL})$ was performed to recontour the left cheek defect, which was successfully augmented at 1 month (B), but substantial absorption was seen at 10 months (C). 

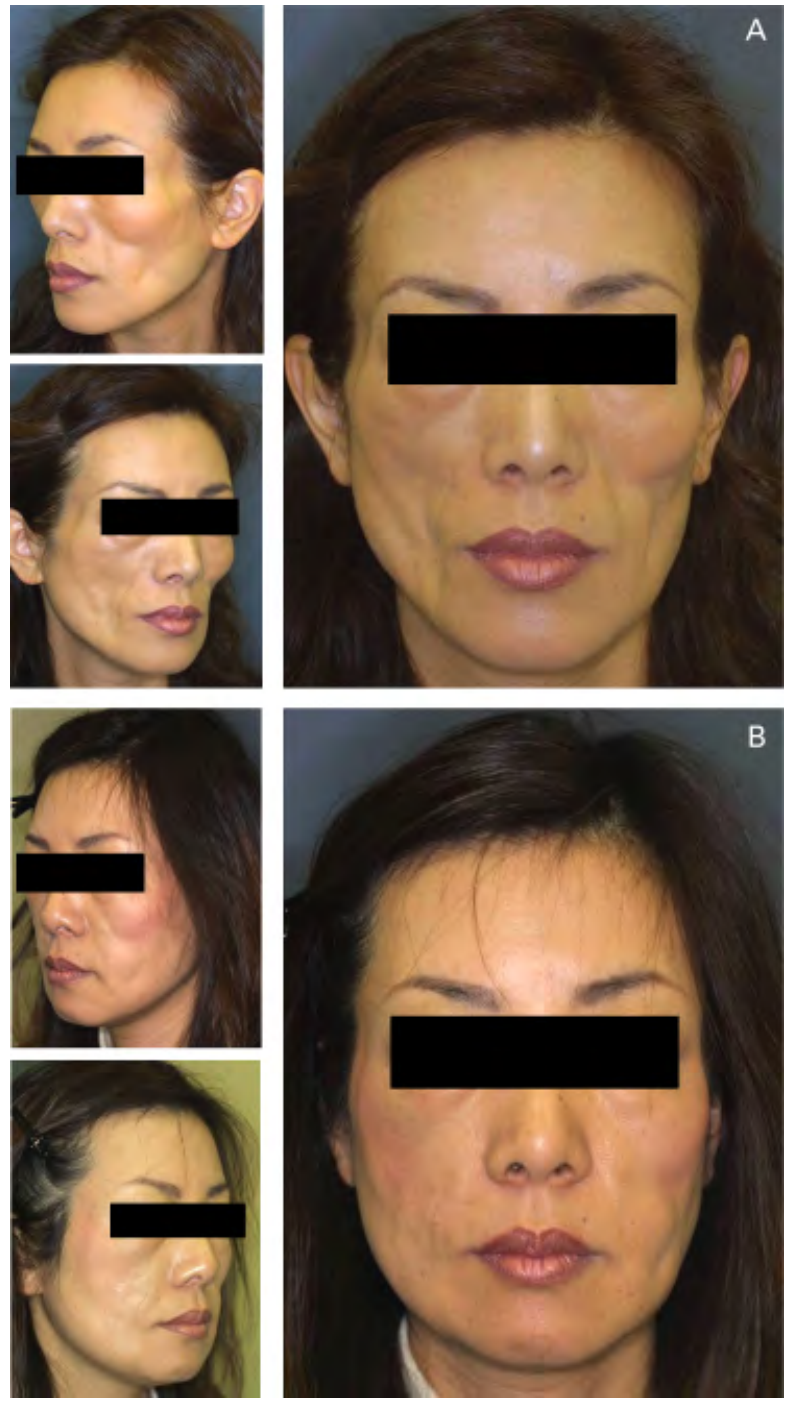

Figure 3. Clinical views of non-CAL Case 2 (a 42-year-old woman): preoperative views (Grade 5 lipoatrophy; A) and postoperative views at 9 months (B). The patient has been taking oral corticosteroid since she was diagnosed with LEP at the age of 20 years. Conventional lipoinjection $(250 \mathrm{~mL})$ was performed to augment facial defects on both cheeks, and she continued the medication perioperatively. Redness and fluctuation were seen on the left cheek at 1 month, and necrotic adipose fluid was removed by a small skin incision. However, the facial defect was relatively improved at 9 months (B).

the number of cells per volume by $25 \%$ for adipocytes and $43 \%$ for ASCs. ${ }^{18}$ We prefer an 18 - or 16-gauge sharp-tipped needle for fat injection because we have found that a sharp needle facilitates placing fat grafts more diffusively and with higher accuracy, even into fibrous or relatively hard tissue and into the muscle through a fascia, compared to a blunt-tipped needle.

Adipose tissue contains not only adipose progenitor cells but also multipotent stem cells that can differentiate into fat, bone, cartilage, and other types of tissue. ${ }^{15,19}$ Previously, we found that aspirated fat loses a significant number of these precursor cells during liposuction with a suction machine and the preparation processes and that aspirated fat has approximately half the number of ASCs as excised whole fat does. ${ }^{16}$ There seems to be two main reasons for the relative deficiency of ASCs. First, a major portion of the ASCs are located around large vessels and are left at the donor site after liposuction; ${ }^{16}$ we have confirmed this phenomenon using histologic analysis (manuscript in preparation). Second, some ASCs are released into the fluid portion of liposuction aspirates. ${ }^{17}$ The resulting relative deficiency of precursor cells in aspirated fat may contribute to the low survival rate and long-term atrophy of transplanted lipoaspirates.

In CAL, we compensate for this ASC deficit by supplementing the aspirated fat intended for lipoinjection with freshly isolated SVF containing ASCs. To maximize the biologic function and avoid unexpected behavior of ASCs, we believe that it is important to ensure adherence of supplemented ASCs to adipocytes or connective tissue. ${ }^{20}$ Centrifugation of adipose grafts seems to be important also for reducing water content in the graft, which may disturb the adherence of supplemented ASCs to the adipose tissue.

Preclinical studies have partly confirmed some potential mechanistic benefits for using ASCs in CAL. ${ }^{16,21,22}$ ASCs can differentiate into adipocytes and contribute to the regeneration of adipose tissue. In addition, ASCs can differentiate into endothelial cells and also probably into vascular mural cells, ${ }^{16,23,24}$ resulting in the promotion of angiogenesis and graft survival. ASCs are known to release angiogenic growth factors in response to hypoxia 
A
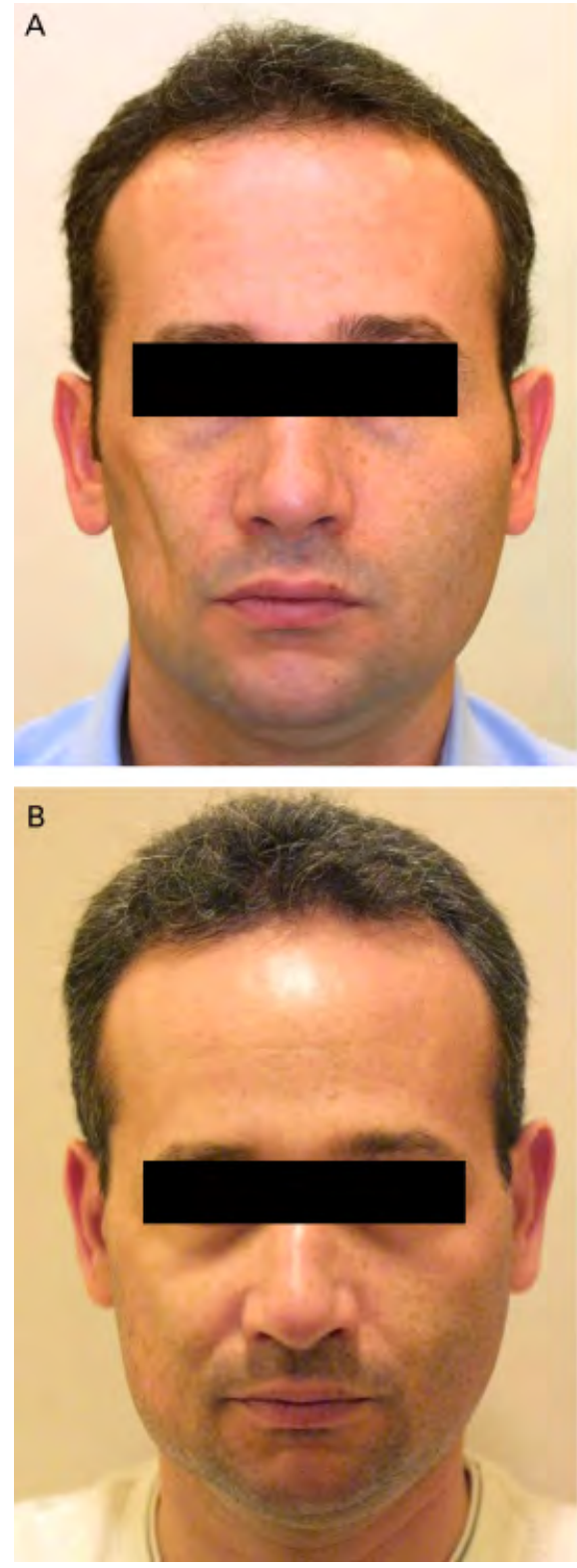
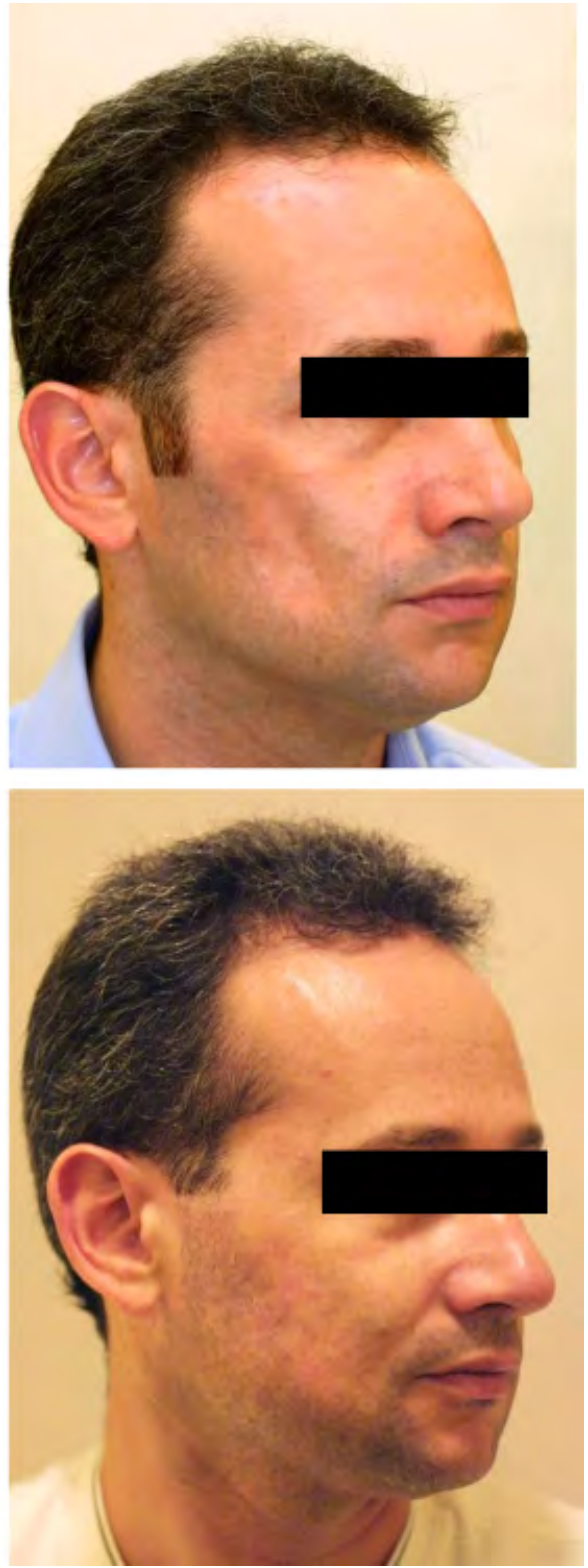

Figure 4. Clinical views of CAL Case 1 (a 35-year-old man): preoperative views (Grade 4 lipoatrophy; A) and postoperative views at 13 months (B). The patient noticed the right facial defect during his $20 \mathrm{~s}$, was diagnosed with PRS, and has no history of oral corticosteroid use. CAL $(110 \mathrm{~mL})$ was performed to correct the facial defect, which was improved, and the facial contour maintained at 13-month follow-up (B). The cheek is soft and natural appearing with no visible scars.

and other conditions, ${ }^{25,26}$ and these factors may promote angiogenesis from surrounding host tissue. The most influential role we considered is that ASCs remain as original undifferentiated ASCs in surviving adipose tissue. ${ }^{16}$ ASCs are assigned to participate in tissue turnover of adipose, and the turnover is known to be very slow, taking 2 years or more. ${ }^{27}$ However, transplanted adipose tissue experiences temporary ischemia followed by reperfusion and so the adipose grafts probably turn over in the early stage (up to 3 months) after transplantation. We hypothesize that the relative deficiency of ASCs in aspirated fat may affect the replacement process and lead to postoperative atrophy of grafted fat, which commonly occurs during the first 6 months after lipoinjection. 

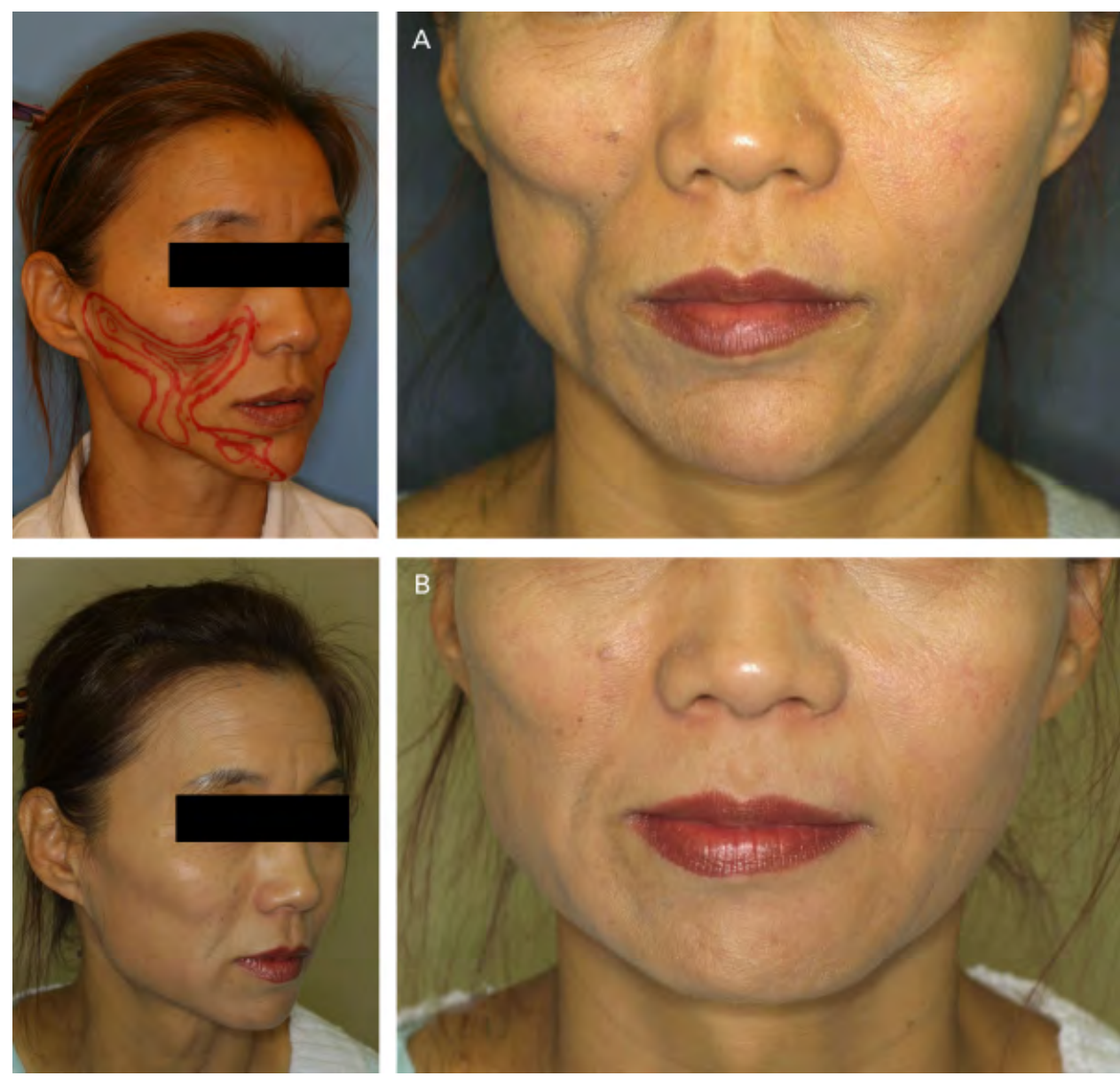

Figure 5. Clinical views of CAL Case 3 (a 48-year-old woman): preoperative views (Grade 4 lipoatrophy; A) and postoperative views at 13 months (B). The patient noticed the right facial defect during her 20s and was diagnosed with LEP. She has a history of oral corticosteroid use, but has not taken them for several years. CAL $(100 \mathrm{~mL})$ was performed to correct the facial defect, which was improved at 9 months follow-up (B).

In our series, we observed no adverse side effects or complications except for one case of lupus erythematosus profundus in the non-CAL group who took oral corticosteroids preoperatively. The patient showed necrosis of a part of the transplanted adipose tissue and the necrotic fluids were drained. A patient in the CAL group who had taken oral corticosteroids showed a lower clinical score compared to non-steroid-taking patients, suggesting that steroids taken perioperatively, or possibly the activity of lupus requiring the medication, has negative effects on angiogenesis and graft survival. Thus, it may be recommended that lipoinjection, either non-CAL or CAL, is performed at a period of minimal lupus activity and after corticosteroid therapy has been discontinued.
There were notable differences between the two groups in the ages of the patients and in the injection volumes in this preliminary trial. Nonetheless, the data suggest that lipoinjection is an effective tool for recontouring facial lipoatrophy, and ASC supplementation is suggested to boost effectiveness. Our clinical experience using CAL for cosmetic breast augmentation with 1 to 4 years of follow-up also suggests that CAL is safe and may be superior to conventional lipoinjection. ${ }^{20}$ We believe that CAL can improve disfigurement and social disability of patients with facial lipoatrophy and that it has some advantages over conventional surgical methods: lack of facial and donor site scarring, lack of complications derived from foreign materials, and 
applicability to any shape of facial defects. Larger studies and longer follow-up times are needed to establish the superiority and the durability of the CAL-mediated improvements in facial lipoatrophy patients.

\section{References}

1. Ascher B, Coleman S, Alster T, et al. Full scope of effect of facial lipoatrophy: a framework of disease understanding. Dermatol Surg 2006;32:1058-69.

2. Sommer A, Gambichler T, Bacharach-Buhles M, et al. Clinical and serological characteristics of progressive facial hemiatrophy: a case series of 12 patients. J Am Acad Dermatol 2006;54:227-33.

3. Burnouf M, Buffet M, Schwarzinger M, et al. Evaluation of Coleman lipostructure for treatment of facial lipoatrophy in patients with human immunodeficiency virus and parameters associated with the efficiency of this technique. Arch Dermatol 2005;141:1220-4.

4. Domergue S, Psomas C, Yachouh J, et al. Fat microinfiltration autografting for facial restructuring in HIV patients. J Craniomaxillofac Surg 2006;34:484-8.

5. Longaker MT, Siebert JW. Microvascular free-flap correction of severe hemifacial atrophy. Plast Reconstr Surg 1995;96:800-9.

6. Vaienti L, Soresina M, Menozzi A. Parascapular free flap and fat grafts: combined surgical methods in morphological restoration of hemifacial progressive atrophy. Plast Reconstr Surg 2005;116:699-711.

7. Carpaneda CA, Ribeiro MT. Percentage of graft viability versus injected volume in adipose autotransplants. Aesthetic Plast Surg 1994;18:17-9.

8. Ersek RA, Chang P, Salisbury MA. Lipo layering of autologous fat: an improved technique with promising results. Plast Reconstr Surg 1998;101:820-6.

9. Ullmann Y, Hyams M, Ramon Y, et al. Enhancing the survival of aspirated human fat injected into nude mice. Plast Reconstr Surg 1998;101:1940-4.

10. Fulton JE, Suarez M, Silverton K, et al. Small volume fat transfer. Dermatol Surg 1998;24:857-65.

11. Sommer B, Sattler G. Current concepts of fat graft survival: histology of aspirated adipose tissue and review of the literature. Dermatol Surg 2000;26:1159-66.

12. Shiffman MA, Mirrafati S. Fat transfer techniques: the effect of harvest and transfer methods on adipocyte viability and review of the literature. Dermatol Surg 2001;27:819-26.

13. Coleman SR. Structural fat grafts: the ideal filler? Clin Plast Surg 2001;28:111-9.

14. Butterwick KJ. Lipoaugmentation for aging hands: a comparison of the longevity and aesthetic results of centrifuged versus noncentrifuged fat. Dermatol Surg 2002;28:987-91.
15. Zuk PA, Zhu M, Ashjian P, et al. Human adipose tissue is a source of multipotent stem cells. Mol Biol Cell 2002;13:4279-95.

16. Matsumoto D, Sato K, Gonda K, et al. Cell-assisted lipotransfer: supportive use of human adipose-derived cells for soft tissue augmentation with lipoinjection. Tissue Eng 2006;12: 3375-82.

17. Yoshimura K, Shigeura T, Matsumoto D, et al. Characterization of freshly isolated and cultured cells derived from the fatty and fluid portions of liposuction aspirates. J Cell Physiol 2006;208:64-76.

18. Kurita M, Matsumoto D, Shigeura T, et al. Influences of centrifugation on cells and tissues in liposuction aspirates: optimized centrifugation for lipotransfer and cell isolation. Plast Reconstr Surg 2008;121:1033-41.

19. Zuk PA, Zhu M, Mizuno H, et al. Multilineage cells from human adipose tissue: implications for cell-based therapies. Tissue Eng 2001;7:211-28.

20. Yoshimura K, Sato K, Aoi N, et al. Cell-Assisted Lipotransfer (CAL) for cosmetic breast augmentation-supportive use of adipose-derived stem/stromal cells. Aesthetic Plast Surg 2008;32:48-55.

21. Masuda T, Furue M, Matsuda T. Novel strategy for soft tissue augmentation based on transplantation of fragmented omentum and preadipocytes. Tissue Eng 2004;10:1672-83.

22. Moseley TA, Zhu M, Hedrick MH. Adipose-derived stem and progenitor cells as fillers in plastic and reconstructive surgery. Plast Reconstr Surg 2006;118(3 Suppl):121S-8S.

23. Miranville A, Heeschen C, Sengenes C, et al. Improvement of postnatal neovascularization by human adipose tissue-derived stem cells. Circulation 2004;110:349-55.

24. Planat-Benard V, Silvestre JS, Cousin B, et al. Plasticity of human adipose lineage cells toward endothelial cells: physiological and therapeutic perspectives. Circulation 2004;109:656-63.

25. Rehman J, Traktuev D, Li J, et al. Secretion of angiogenic and antiapoptotic factors by human adipose stromal cells. Circulation 2004;109:1292-8.

26. Kilroy GE, Foster SJ, Wu X, et al. Cytokine profile of human adipose-derived stem cells: expression of angiogenic, hematopoietic, and pro-inflammatory factors. J Cell Physiol 2007; 212:702-9.

27. Strawford A, Antelo F, Christiansen M, et al. Adipose tissue triglyceride turnover, de novo lipogenesis, and cell proliferation in humans measured with $2 \mathrm{H}_{2} \mathrm{O}$. Am J Physiol Endocrinol Metab 2004;286:E577-88.

Address correspondence and reprint requests to: Kotaro Yoshimura, MD, Department of Plastic Surgery, University of Tokyo School of Medicine; 7-3-1 Hongo, Bunkyo-ku, Tokyo 113-8655, Japan, or e-mail: yoshimura-pla@ h.u-tokyo.ac.jp 